highlighted that most evidence about the use of PROMs in palliative care populations is in the outpatient oncology setting. There is little evidence about the completion of PROMs in the inpatient palliative care setting. St Christopher's Index of Patient Priorities (SKIPP) 2 is a validated questionnaire exploring symptoms and quality-of-life and given to all patients on their third and tenth day of hospice stay. The completion of one SKIPP on the third day has been used as a PROM as it enquires about the patients' symptoms and quality of life prior to, and after contact/admission to the hospice service.

Methods A retrospective analysis from 150 consecutive admissions lasting four or more days across two hospice sites in the South West of England to determine the completion rates of SKIPP.

Results Of 150 admissions, 61 stayed less than 10 days (and thus expected to complete one SKIPP) and 89 stayed longer than 10 days (and thus expected to complete two SKIPPs). One SKIPP form was completed by $57.3 \%$ of all patients. Of the patients who stayed for 10 days or greater, $33.7 \%$ completed a second SKIPP form. The most common reasons for non-completion were the patient was too unwell $(44.3 \%)$, or the form was not able to be completed on day three $(21.4 \%)$ and therefore deemed an inappropriate outcome measure.

Discussion Nearly $60 \%$ of patients managed to complete one SKIPP questionnaire which can be used alone as a PROM for patients in an inpatient palliative care setting. This suggests PROM can be routinely embedded into clinical practice within a hospice setting.

\section{P-79 REMOVING TECHNICAL BARRIERS: IPOS TIMELINE TOOL AND IPOS REPORT WITH ST BARNABAS HOSPICE AND LOROS}

Sean Leathen. Leathen Healthcare IT Solutions, Bolton, UK

\subsection{6/bmispcare-2017-hospice.106}

Aims Two tools are now in use across St Barnabas and LOROS sites, which support clinicians in getting the best outcomes from IPOS and OACC measures. These are the IPOS Timeline tool and IPOS Report. The IPOS Timeline tool generates a series of graphs about a single patient. The IPOS Report provides a comprehensive assessment of a service's performance for a given time frame. These aim to help services to make the best use of all their IPOS data, by automating processes and removing any technical barriers.

Results The IPOS Timeline has been designed for clinicians. By pressing a single button in a SystmOne extract, it creates a set of graphs showing all IPOS information about the patient. Using this, the clinician can see trends, current and past problems at a glance. This saves time with the patient, or in explaining the patient's history to other clinicians. The IPOS Report has automated the task of analysing all IPOS data across an entire organisation. It uses this information to create an in depth report of service performance, and a full suite of graphs and measures for each IPOS indicator. No data analysis or advanced computer skills are required. The IPOS Report shows where teams are performing well and what can be improved. It contains graphs of service trends over time, and visualises the current level of patient care and how patients needs are changing. A summary shows all this at service level, with a simple rating system. Together, these tools have removed the barrier of technical knowledge and analytical skills needed to handle large amounts of IPOS data. This has enabled services to become more responsive and improve their delivery of patient care.

\section{P-80 SCOPING THE LOCAL LANDSCAPE OF END OF LIFE CARE WITH ROUTINE DATA AND QUALITATIVE RESEARCH}

'Lynn Sudbury-Riley, 'Phillipa Hunter-Jones, 'Jonathan Moss, '2ucy Smith. 'University of Liverpool, Liverpool, UK; ${ }^{2}$ Oakhaven Hospice, Lymington, UK

\subsection{6/bmjspcare-2017-hospice.107}

Background Hospices need to engage in research to ensure provision of the highest possible quality of palliative and end of life care for patients and families. A hospice collaborated with researchers at a world-leading university on a research project to scope the current landscape of palliative and end of life care needs within their geographical area. A postdoctoral research fellow was recruited in May 2017 to conduct and deliver the research over a period of six months.

Aim(s) The research aims to identify what is happening in the hospice's catchment area, and establish why these trends are happening.

Methods Routinely collected health and social care data provide an efficient and useful opportunity for evaluating and improving palliative and end of life care services (Davies et al., 2016). Data from ONS, Public Health England and the primary care network will be used to establish:

- How many people have died in the hospice's catchment area since 2014

- What these people died from

- How many of these deaths did and did not have hospice involvement.

Empirical data collected via qualitative and quantitative research tools will then be used to understand why these trends are happening. Data will be collected from multiple stakeholders and then subjected to systematic thematic analysis. Stakeholders include:

- Health care professionals $(n=20)$

- Hospice service users $(n=20)$

- Non-hospice users $(n=20)$

- Wider population (questionnaires accessed via GP practices).

Results Once the research is complete, these findings will inform the hospice's clinical strategy by providing robust evidence of where direction, activity and resources are most needed. It is anticipated the project will also generate future opportunities for the hospice and research team to develop practical and academic outputs.

Conclusions Hospices and universities can work in partnership to learn about the landscape of end of life care needs in different localities using routine data and qualitative research.

\section{P-81 LEARNING TO FLY: EXPERIENCES OF A FLEDGLING RESEARCH HOSPICE}

Helen Birch. Queenscourt Hospice, Southport, UK

10.1136/bmjspcare-2017-hospice. 108 